\title{
MicroFit: um software gratuito para desenvolvimento e ajuste de modelos matemáticos de crescimento bacteriano
}

\author{
MicroFit: a free software for the development and fit \\ of mathematical models for bacterial growth
}

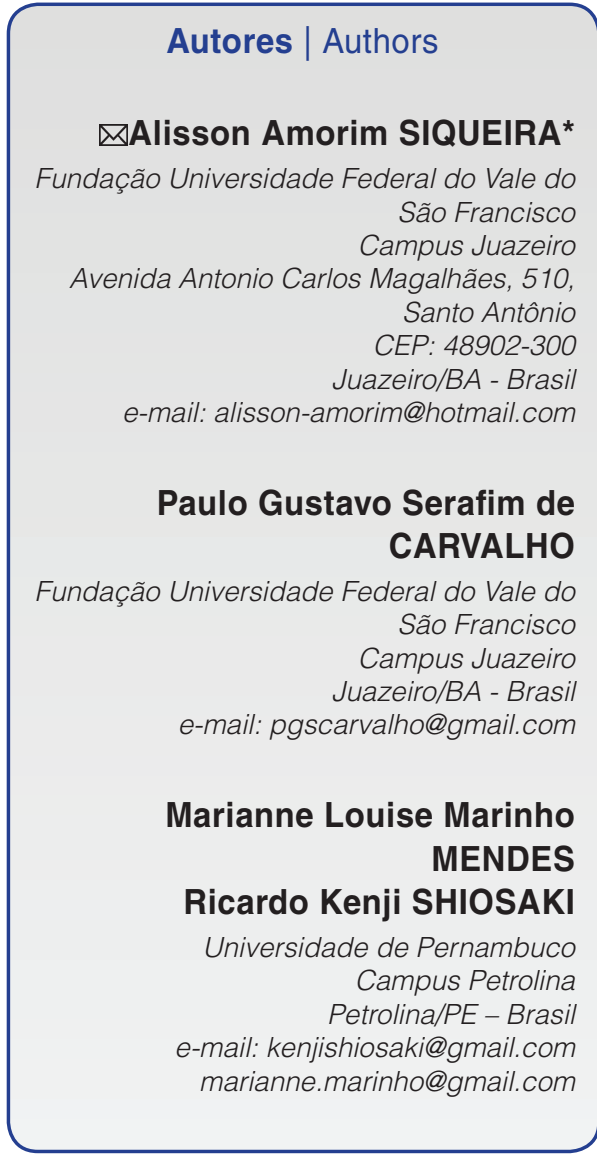

$\triangle$ Autor Correspondente / Corresponding Author

\section{Resumo}

A conservação de alimentos é uma das práticas mais antigas apropriadas pela humanidade, entretanto as infecções alimentares ainda despontam como uma gravíssima questão de saúde pública em todo o mundo, levando a óbito milhares de pessoas todos os anos. Nesse contexto, os modelos de predição microbiológica surgem como uma importante ferramenta para aferição da segurança alimentar e avaliação do risco de contaminação de alimentos. Os parâmetros de predição do comportamento microbiano proporcionam um caminho rápido e relativamente econômico para obtenção de estimativas confiáveis sobre crescimento, inativação e sobrevivência durante a atividade de micro-organismos nos alimentos, de acordo com as condições de estocagem. Apesar da importância dos modelos de predição, as dificuldades de utilização e resolução dos mesmos, por consistirem de equações não lineares e sem solução analítica, impedem sua ampla utilização. A baixa disponibilidade de programas eletrônicos para análise de dados microbiológicos, bem como a falta de ferramentas que permitam a criação de modelos ou o ajuste de parâmetros de uma forma rápida e fácil são outros entraves ao uso dos modelos de crescimento microbiológico. Diante desse problema, esse artigo tem por objetivo descrever o processo de desenvolvimento de um software concebido para permitir o ajuste, criação e comparação de modelos microbiológicos de crescimento de bactérias. A partir do uso de ferramentas comumente empregadas nesse tipo de estudo e das necessidades não contempladas por elas, foi desenvolvido um programa autônomo, gratuito, dotado de uma interface objetiva e com mecanismos capazes de criar e avaliar facilmente modelos preditivos. Espera-se que o software desenvolvido neste trabalho possa facilitar a análise de dados do comportamento microbiano em alimentos e o controle de possíveis repercussões para o consumidor final.

Palavras-chave: Modelos de predição microbiológica; Infecções alimentares; Software aplicado à microbiologia. 


\section{Summary}

Food preservation is one of the oldest practices adopted by humanity, however foodborne infections still emerge worldwide as an extremely serious public health issue, leading to the death of thousands of people every year. Thus microbiological prediction models emerge as an important tool for the assessment of food safety and the risk of foodborne infections. Microbial behavior prediction parameters provide a quick and relatively economical way of obtaining reliable estimates of the growth, inactivation and survival of microorganisms during their activity in food, according to the storage conditions. Despite the importance of prediction models, the difficulties involved in their resolution, due to the fact that they consist of non-linear equations with no analytical solution, prevent their widespread use. The limited availability of electronic programs for the analysis of microbiological data, and the lack of tools that allow for the quick and easy creation of models or the fit of parameters are other barriers to the use of microbiological growth models. Faced with this situation, the objective of this study was to describe the process of developing software designed to allow for the fit, creation and comparison of microbiological models for bacterial growth. As from the use of widely used tools for this type of study and the needs not covered by them, a free stand-alone program, was developed with an objective user interface and mechanisms capable of creating and easily evaluating predictive models. It is hoped that the software developed in this work can facilitate the analysis of the data of microbial behavior in food and the control of possible repercussions for the final consumer.

Key words: Predictive microbiological models; Foodborne infections; Software applied to microbiology. 


\section{Introdução}

A importância da alimentação para a sobrevivência humana tornou a conservação de alimentos uma das primeiras práticas apropriadas pela humanidade (MOREIRA, 2010). Conservar um alimento significa reduzir a atividade de micro-organismos a fim de manter as características físicas, químicas e biológicas de um produto por mais tempo, controlando a quantidade de populações microbianas que possam provocar doenças e infecções alimentares (VALSECHI, 2006).

Apesar do consenso de que os alimentos devem ser conservados e estocados corretamente, as infecções alimentares são uma gravíssima questão de saúde pública em todo o mundo. Anualmente, nos Estados Unidos, ocorrem 48 milhões de casos, 128 mil internações e 3 mil óbitos relacionados a Doenças Transmitidas por Alimentos (DTAs), segundo os Centros de Controle e Prevenção de Doenças (CDC, 2011). No Brasil, o Sistema de Informação sobre Mortalidade (SIM) calcula que de 1999 a 2002 ocorreram 25.281 óbitos por doenças transmitidas por alimentos, com uma média de 6.320 óbitos/ano (BRASIL, 2005).

A conservação de alimentos e a manutenção das características físicas e químicas dos produtos são uma das áreas de estudo da microbiologia preditiva. Essa ciência estuda como as propriedades dos alimentos podem ser afetadas pela quantidade de micro-organismos e pelas condições de estocagem, através de modelos e equações matemáticas (CASTRO et al., 2007; NAKASHIMA et al., 2000). O aumento acentuado da preocupação pública com o fornecimento de alimentos microbiologicamente seguros e saudáveis impulsionou o desenvolvimento da microbiologia preditiva, sobretudo o desenvolvimento de modelos de predição microbiológica (TELEKEN et al., 2011).
Os modelos de predição são definidos por Arroyo-López et al. (2014) como uma das mais importantes ferramentas para aferição da segurança alimentar e avaliação de risco nos alimentos, pois, através das curvas de crescimento, o pesquisador poderá explicar como ocorre a proliferação de um micro-organismo em determinado alimento, prever o tempo que esse alimento pode permanecer na prateleira, maximizar esse tempo pela modificação das condições de estocagem, como a temperatura, e informar ao consumidor até quando é possível utilizar o produto sem riscos.

Além de permitir a investigação e avaliação dos processos de conservação de alimentos e produtos, os modelos preditivos proporcionam um caminho rápido e relativamente econômico para obtenção de dados confiáveis sobre crescimento, inativação e sobrevivência durante o desenvolvimento microbiano (SARMENTO, 2006).

Um modelo preditivo pode ser de nível primário, quando mede a resposta de um micro-organismo no tempo para um ambiente de condições estáticas, a exemplo de uma câmara com temperatura fixa, de nível secundário, quando descreve a resposta dos parâmetros do modelo primário para as variações das condições ambientais, como em uma câmara simulando uma variação de temperatura em diversos pontos de uma rota de distribuição, ou de nível terciário, quando se trata de software ou solução eletrônica para resolver os modelos do nível primário ou secundário (SWINNEN et al., 2004).

Os modelos preditivos primários são em sua maioria equações não lineares, a exemplo do modelo de Gompertz modificado (Equação 1) e do modelo de Baranyi-Roberts (Equação 2), enquanto que os modelos secundários são equações diferenciais, a exemplo da equação de Van Impe (Equação 3), conforme apresentados na Tabela 1.

Tabela 1. Exemplos de modelos de predição microbiológica e suas equações.

\section{Modelo}

Equação

Equação Gompertz

(ZHOU et al., 2012)

Baranyi e Roberts

(CAYRÉ et al., 2007)

Van Impe (Gompertz)

Modelo secundário

(HUANG, 2003)

$$
y(t)=A \cdot e^{\left[-e^{-B(t-\mu)}\right]}
$$

$$
y(t)=y f+\ln \left[\frac{-1+e^{\mu \max . \lambda}+e^{\mu \max . t}}{-1+e^{\mu \max . t}+e^{\mu \max . \lambda+y f-y 0}}\right]
$$

$$
\frac{d y}{d t}=\mu(t)[y(t)-A] \cdot \ln \left[\frac{B-A}{y(t)-A}\right] \text {, }
$$

$$
\mu(t)=a(T-T \min )^{2}\left[1-e^{b(T-T \max )}\right]
$$


Nos modelos da Tabela 1 " $y(t)$ " representa a quantidade de colônias em um instante de tempo "t"; "e" representa a função exponencial; "In" o logaritmo natural e "A", "B", " $\mu$ ", " $y_{f}$ ", " $\mu_{\max }$ ", " $\lambda$ ", " $y_{0}$ ", "a" e "b" são parâmetros que podem ser modificados para determinar o grau de ajuste dos modelos; "dy/dt" representa a primeira derivada da quantidade de colônias em relação ao tempo; e " $\mu(t)$ " é a taxa de crescimento na temperatura "T", variante no tempo.

Trabalhar com equações não lineares e diferenciais desse tipo implica em dispêndio de considerável tempo e atenção, pois uma pequena variação em um dos parâmetros provoca uma grande mudança no valor final da função. Além disso, a aplicação do método dos mínimos quadrados para o ajuste dos dados experimentais resulta em um sistema de equações não lineares, sem solução analítica, sendo necessário recorrer a métodos numéricos e computacionais, por vezes inacessíveis aos pesquisadores fora da área de exatas (SWINNEN et al., 2004). A estimativa dos parâmetros ainda depende da indicação de um valor inicial próximo da solução real, para permitir que algoritmos possam calcular os parâmetros corretamente. Se a indicação for distante da solução, o algoritmo não irá convergir e gerar a resposta esperada, forçando o pesquisador a experimentar soluções por tentativa e erro/acerto. Os entraves apresentados tornam-se ainda mais difíceis ao se utilizarem modelos de nível secundário, com equações diferenciais, que além do ajuste intrínseco a esses modelos, ainda necessitam de integração numérica.

A dificuldade de utilização dos modelos primários e secundários, torna essencial o uso de um modelo de nível terciário, uma motivação para o desenvolvimento de softwares que busquem facilitar o ajuste de dados experimentais aos modelos de crescimento teórico, o que pode ser de utilidade para pesquisadores e empresas (GEERAERD, 2005).

Atualmente estão disponíveis pouquíssimos programas e ferramentas desenvolvidas com intuito de trabalhar com modelos de predição. As poucas soluções existentes geralmente não possuem executáveis autônomos, rodam apenas dentro de um programa proprietário, a exemplo do GInaFit e DMFIT, que funcionam como complementos do Microsoft ${ }^{\oplus}$ Excel $^{\mathrm{TM}}$ (ARROYO-LÓPEZ et al., 2012), apresentam dependências externas, baixa curva de aprendizado e todas as soluções executam apenas modelos internos, não permitindo criação de modelos ou adição de novos parâmetros (como pH, atividade de água ou concentração de sal) aos modelos existentes.

Algumas soluções, como PMP, SSSP e alternativas comerciais (MCCLURE; AMÉZQUITA, 2009), possuem executáveis, contudo não possibilitam a criação de modelos e incorporação de novos parâmetros, ficando restritos aos principais modelos encontrados na literatura e a um grupo reduzido de alimentos, tornando trabalhoso e demorado novos esforços para desenvolver, testar e validar modelos preditivos.

Diante da importância da microbiologia para a qualidade e segurança dos alimentos, bem como para o desenvolvimento econômico e social ao qual essa ciência está intrinsecamente relacionada, é de grande importância a criação e validação de modelos cada vez mais precisos para determinação de métricas relacionadas às condições de armazenagem de alimentos, entretanto os softwares disponíveis atualmente não dão suporte a essa tarefa.

Este trabalho tem por objetivo descrever o processo de desenvolvimento de um software gratuito, autônomo e de fácil utilização voltado para o ajuste, criação e comparação de modelos microbiológicos de crescimento de bactérias. Espera-se que o software descrito neste trabalho possa facilitar a análise de dados do comportamento microbiano em alimentos e incentivar o uso de modelos de predição.

\section{Materiais e métodos}

Inicialmente foi realizado um levantamento dos softwares descritos na literatura e em compilações on-line, como ferramentas capazes de ajustar dados a modelos primários ou secundários (ARROYO-LÓPEZ et al., 2012, 2014; PMM, 2014; UC FOOD SAFETY, 2014). As características úteis, funções ausentes e as falhas de cada software foram registradas em um checklist de funcionalidades, com soluções para os problemas encontrados. As características foram obtidas com base no uso dos softwares, descrições dos desenvolvedores e documentação dos programas identificados.

Após a compilação da checklist, foram planejadas as funcionalidades de um software que atendesse aos critérios da lista compilada, através de protótipos em folhas de papel, de forma que o usuário final pudesse acessar cada ferramenta adicionada de forma fácil e intuitiva. Dados reais de crescimento bacteriano, para teste e validação do programa, foram obtidos gratuitamente no site do Combase (www.combase.cc), uma base de dados on-line com grande quantidade de respostas para crescimento de micro-organismos em diversos alimentos, administrada conjuntamente pelo Institute of Food Research (UFR), no Reino Unido, pelo USDA Agricultural Research Service (USDA-ARS), nos Estados Unidos, e pela University of Tasmania Food Safety Centre (FSC), na Austrália (TELEKEN et al., 2011; COMBASE, 2014). A codificação da aplicação foi realizada na linguagem $\mathrm{C}++$, dentro do ambiente de desenvolvimento integrado C++ Builder 2007, no Sistema Operacional Windows 7. 
Inicialmente foram programadas as rotinas de leitura e inserção de dados, como importação de arquivos de planilha (.csv e documentos do Microsoft ${ }^{\circledR}$ Excel $^{T M}$ ) e digitação de dados pelo usuário. Independente do formato, a leitura dos dados espera receber um arquivo com múltiplas colunas, cada uma representando um parâmetro do modelo a ser utilizado (tempo, pH, atividade de água, concentração de sal etc.). Optou-se por armazenar internamente ao programa os dados digitados e importados pelo usuário.

Após desenvolver os procedimentos de leitura de dados, foram codificadas as rotinas para geração de estatísticas de dispersão dos dados armazenados, que comparam o valor observado em laboratório e o valor estimado pelos modelos do programa, além das estatísticas de comparação, que permitem ao usuário decidir qual o melhor modelo. Entre os dados estatísticos programados estão média, desvio padrão, coeficiente de correlação de Pearson, erro médio relativo, erro médio estimado e dados residuais e totais.

Para superar a ausência de softwares que permitissem o desenvolvimento de novos modelos, sejam eles primários ou secundários, foi desenvolvido um módulo no qual os usuários pudessem criar seus próprios modelos, digitando unicamente a equação desejada, por meio de funções matemáticas como exponenciais, potenciação, logaritmos, funções trigonométricas e funções comuns em planilhas eletrônicas.

A complexidade de avaliar uma equação genérica digitada pelo usuário pôde ser superada através da criação de um interpretador de expressões matemáticas, composto pelos quatro blocos funcionais de um reconhecedor de linguagens computacionais: o leitor de símbolos (Scanner), o organizador e identificador de sintaxe (Parser), a árvore de sintaxe abstrata (Abstract Syntax Tree) e o avaliador (Evaluator).

Após a construção do interpretador, foram implementadas as rotinas para ajuste automático dos dados, como o algoritmo de Levenberg-Marquardt (GAVIN, 2013), responsável por avaliar os valores de parâmetros para o ajuste de modelos, e as rotinas para resolução de equações diferenciais, pelo método de Runge-Kutta $4^{a}$ ordem (VALLE, 2012). Ambos os métodos foram testados e validados dentro do Open Office 4.0.1 $1^{\mathrm{TM}}$, através do pacote para resolução de equações não lineares Solve ${ }^{\mathrm{TM}}$ (SOLVER, 2014).

Após a codificação das rotinas de processamento de dados, a interface gráfica do software foi desenhada, com o objetivo de proporcionar uma familiarização do usuário com o programa desde o primeiro uso, além de possibilitar a importação e digitação rápida de dados, visualização gráfica das informações inseridas no programa, criação, avaliação e modificação de modelos preditivos, ajuste automático de parâmetros e exportação dos resultados gerados. As rotinas desenvolvidas foram validadas com dados provenientes do Combase, em sua maioria dados de leite e carne.

\section{Resultados e discussão}

Antes de se iniciar o desenvolvimento do software foram analisadas soluções citadas na literatura especializada e em compilações web (ARROYO-LÓPEZ et al., 2014; PMM, 2014; UC FOOD SAFETY, 2014) cuja função principal fosse a predição microbiológica. Dentre as soluções citadas pelos autores estão ComBase Predictor, DMFit 3.0, GInaFit, PMP 7.0 (Pathogen Modeling Program), SSSP 3.0 (Seafood Safety and Spoilage Predictor), Bugdeath 1.0, FSP (Food Spoilage Predictor), PMM Lab (Predictive Microbial Modeling plug-in for KNIME) e IPMP 2013 (Integrated Pathogen Modeling Program). A Tabela 2 lista algumas das qualidades e deficiências encontradas na análise preliminar.

Dentre as desvantagens encontradas nos softwares testados destacam-se também a dependência de pacotes e programas proprietários, como o Excel ${ }^{\mathrm{TM}}$, a falta de executáveis autônomos, a interface pouco intuitiva, a baixa curva de aprendizado, a impossibilidade de importar ou trabalhar com dados externos aos programas, ausência de funções para exportar dados, ferramentas voltadas para modelos e micro-organismos específicos, falta de suporte multilíngue, impossibilidade de desenvolver novos modelos, adicionar, remover parâmetros ou trabalhar em situações além das preestabelecidas pelo desenvolvedor. Apesar dos problemas encontrados, as soluções disponíveis apresentam uma diversificada e rica base de dados sobre diversos micro-organismos, situação e ambientes e algumas soluções permitem trabalhar com modelos primários e secundários.

Com intuito de sanar os problemas identificados e citados anteriormente, cada ferramenta do software intitulado MicroFit foi cuidadosamente planejada, sobretudo a interface gráfica do usuário. Através de uma interface simples, com menus claros e diretos (Figura 1), o usuário já no primeiro acesso consegue localizar a

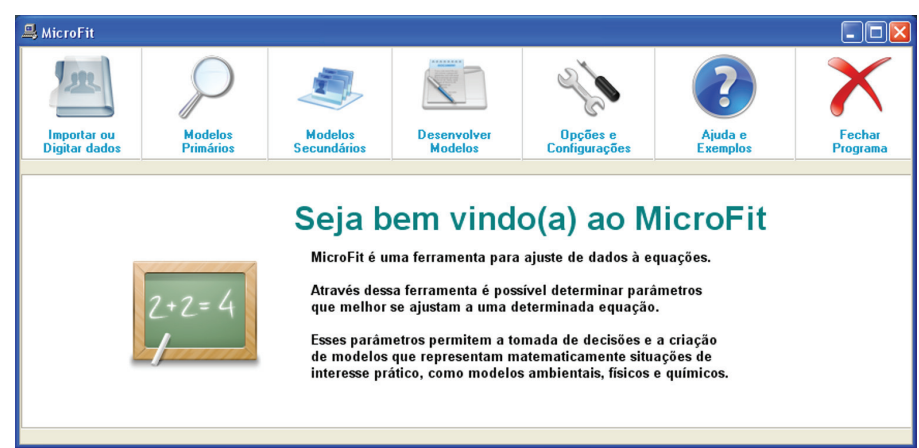

Figura 1. Menu principal do software MicroFit. 
Tabela 2. Qualidades e deficiências dos programas identificados.

\begin{tabular}{|c|c|c|}
\hline Programa & Qualidades & Deficiências \\
\hline DMFit/GInaFIT & $\begin{array}{l}\text { Ajuste de dados de modelos primários e } \\
\text { secundários }\end{array}$ & $\begin{array}{l}\text { Dependência do Excel, baixa curva de } \\
\text { aprendizado e interface confusa }\end{array}$ \\
\hline ComBase Predictor & Interface amigável, on-line, fácil e rápido & $\begin{array}{l}\text { Modelos fixos, sem possibilidade de } \\
\text { extrapolação das equações }\end{array}$ \\
\hline SSSP & $\begin{array}{l}\text { Programa rico em exemplos, com } \\
\text { predição do tempo de prateleira e } \\
\text { suporte multilíngue }\end{array}$ & $\begin{array}{l}\text { Interface não amigável, modelos fixos, } \\
\text { problemas de compatibilidade por conta } \\
\text { do Framework de programa utilizado }\end{array}$ \\
\hline FSP & $\begin{array}{l}\text { Facilidade para trabalhar com perfis de } \\
\text { temperatura }\end{array}$ & $\begin{array}{l}\text { Uso específico para Pseudomonas e } \\
\text { funciona apenas com data logger pago }\end{array}$ \\
\hline Bugdeath 1.0 & $\begin{array}{l}\text { Possui modelos de inativação com } \\
\text { transferência dinâmica de calor }\end{array}$ & $\begin{array}{l}\text { A funcionalidade é restrita para uma } \\
\text { pequena quantidade de problemas }\end{array}$ \\
\hline PMM Lab & $\begin{array}{l}\text { Possui modelos de inativação, } \\
\text { crescimento e sobrevivência que podem } \\
\text { ser utilizados por diagramas }\end{array}$ & $\begin{array}{l}\text { O programa é uma extensão do } \\
\text { Konstanz Information Miner, com } \\
\text { interface confusa à primeira vista }\end{array}$ \\
\hline PMP 7.0 & $\begin{array}{l}\text { Possui modelos de sobrevivência, } \\
\text { crescimento e inativação para vários } \\
\text { micro-organismos e condições }\end{array}$ & $\begin{array}{l}\text { Interface pouco intuitiva e mesmo com } \\
\text { as inúmeras condições de uso não é } \\
\text { possível extrapolar o escopo }\end{array}$ \\
\hline IPMP 2013 & $\begin{array}{l}\text { Diversos modelos primários e } \\
\text { secundários de crescimento e } \\
\text { inativação, com uso fácil e rápido }\end{array}$ & $\begin{array}{l}\text { O ajuste de dados ignora os parâmetros } \\
\text { internos dos modelos e é conduzido } \\
\text { manualmente pelo usuário }\end{array}$ \\
\hline
\end{tabular}

ferramenta desejada. Em cada um dos menus é possível encontrar exemplos, dicas e formas de uso, além de funções para exportação de dados. Apresentado nesse trabalho em Português do Brasil, o software pode ser traduzido para outras línguas dentro do menu Opções e configurações, tendo como idioma padrão o inglês.

A interface para entrada de dados (Menu importar ou Digitar dados) é igualmente intuitiva. Através de células semelhantes às encontradas em planilhas eletrônicas, o usuário pode digitar, importar arquivos, filtrar os dados disponíveis, adicionar e remover colunas (que representam os parâmetros), exportar os dados em visualização e aplicar modelos primários e secundários aos dados salvos no programa, além de trabalhar com múltiplos perfis de dados e modelos simultaneamente (Figura 2a).

Os dados utilizados para testar e validar o programa foram obtidos gratuitamente no site do Combase, contendo a concentração de micro-organismos em função do tempo, durante as fases de crescimento microbiano, como representado na Figura 2b.

Para utilizar um modelo primário, o pesquisador precisa apenas selecionar o perfil de dados e o modelo desejado, fazendo com que o MicroFit processe o pedido e exiba o resultado do ajuste e dos valores dos parâmetros instantaneamente, sem a necessidade de conhecimentos matemáticos ou indicação de valores iniciais para os ajustes, como apresentado na Figura 3.

Os modelos de nível secundário, que incorporam a mudança de condições ambientais dentro dos modelos primários, estão presentes no software e permitem e incorporação de perfis de temperatura ou quaisquer outros parâmetros que afetem a taxa de crescimento (Figura 4). As equações diferenciais são resolvidas pelo método de Runge-Kutta, através de aproximação numérica.

Além de permitir a incorporação de perfis dinâmicos de temperatura ou qualquer outra variável que afete o crescimento, através de importação de planilhas ou digitação de dados, é possível mudar as funções de efeitos do crescimento dinâmico, como equações de Arrhenius e modelo da raiz quadrada (NAKASHIMA et al., 2000).

Os dados inseridos pelo usuário podem ser utilizados em ajustes teóricos de crescimento microbiano de nível primário e secundário, permitindo que o pesquisador estime o tempo de prateleira, parâmetros de inativação e outras possibilidades condizentes com os parâmetros adicionados, de forma muito mais fácil e flexível do que utilizando as ferramentas atuais.

A análise estatística, como o erro médio, grau de ajuste, influência dos parâmetros e as ferramentas de comparação entre modelos permitem a desambiguação de resultados e modelos, além de fornecer métricas confiáveis para as escolhas realizadas. Para fornecer essas métricas, o programa desenvolvido faz uso do algoritmo de Levenberg-Marquardt, que se diferencia de outras técnicas de minimização de erro pelo fato de não necessitar de uma indicação inicial próxima do valor verdadeiro, como o método de Newton (VALLE, 2012). 
MicroFit: um software gratuito para desenvolvimento e ajuste de modelos matemáticos de crescimento bacteriano SIQUEIRA, A. A. et al.

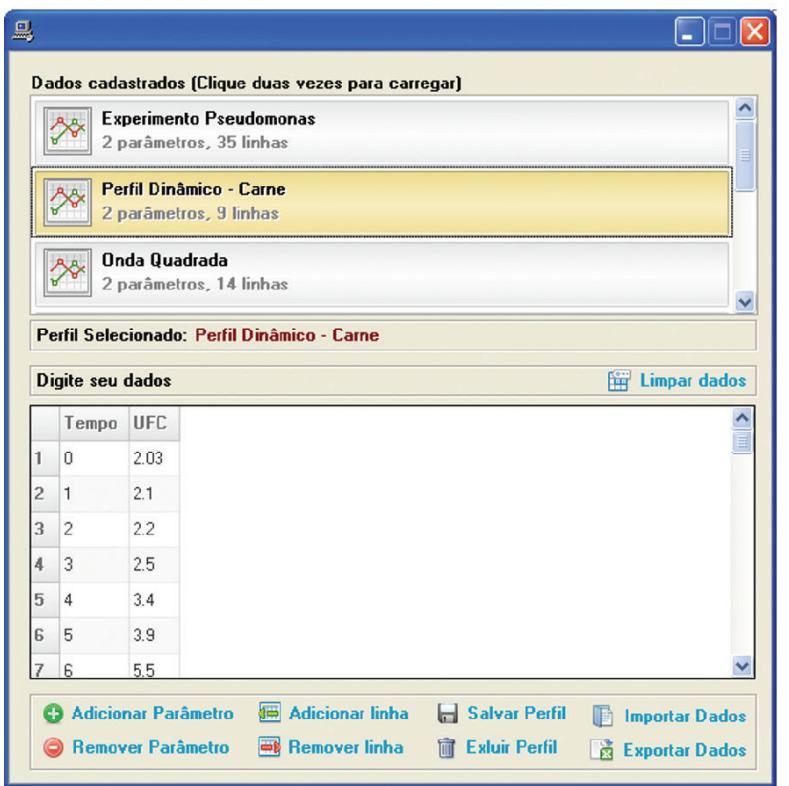

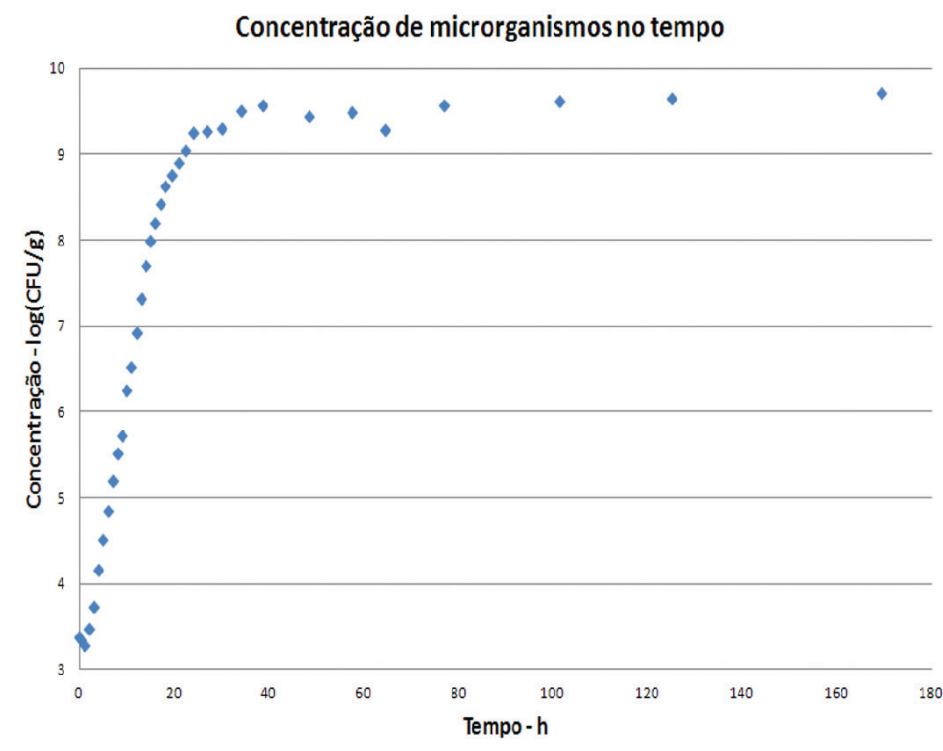

Figura 2. (a) Interface de entrada de dados; (b) exemplo de dados de Pseudomonas ssp. em leite de vaca, obtidos no Combase.

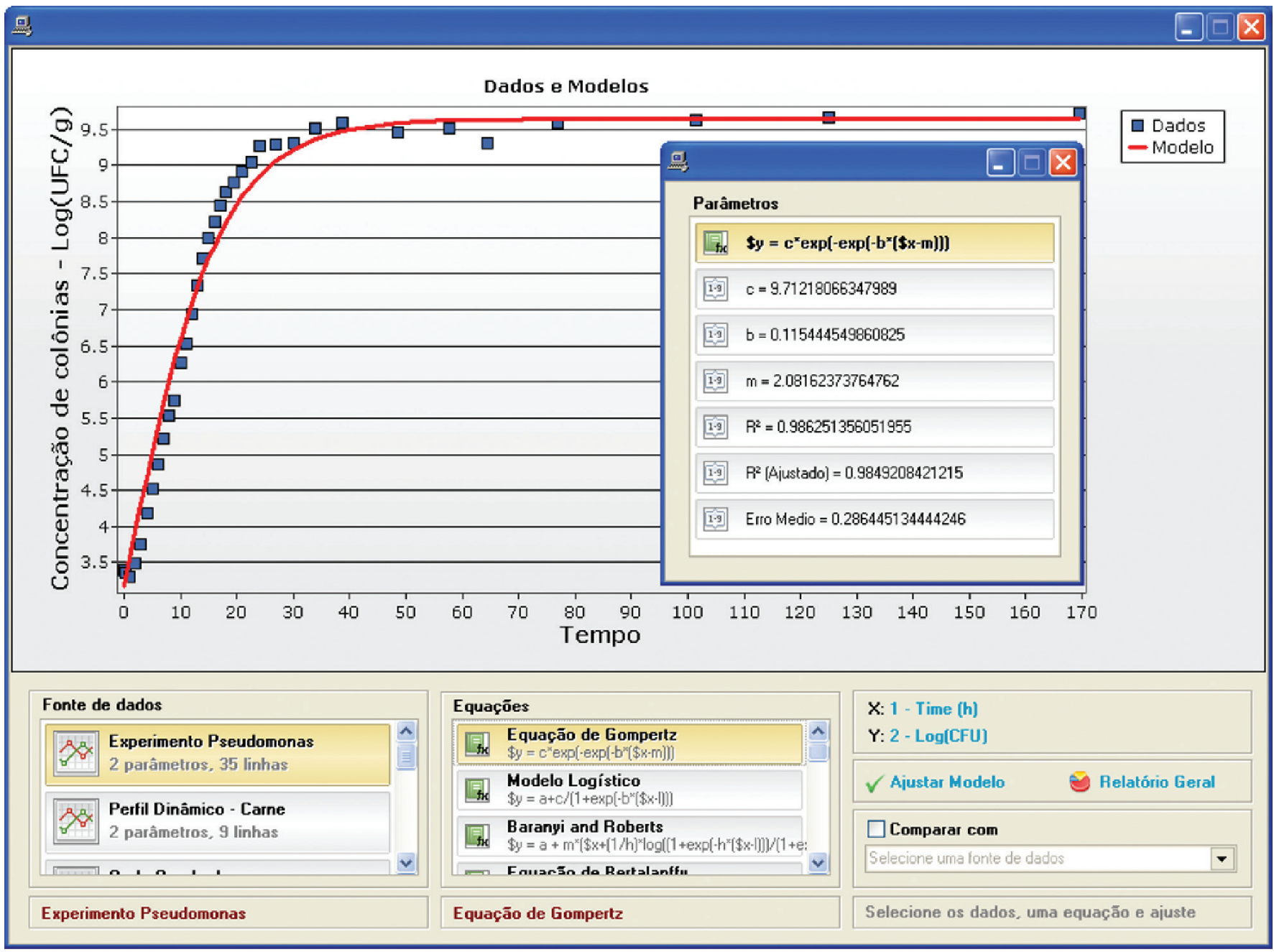

Figura 3. Aplicação de modelos primários (linhas) para ajuste de dados teóricos (pontos), com eixo x representado o tempo e eixo y a quantidade de colônias, em log (UFC/g). 


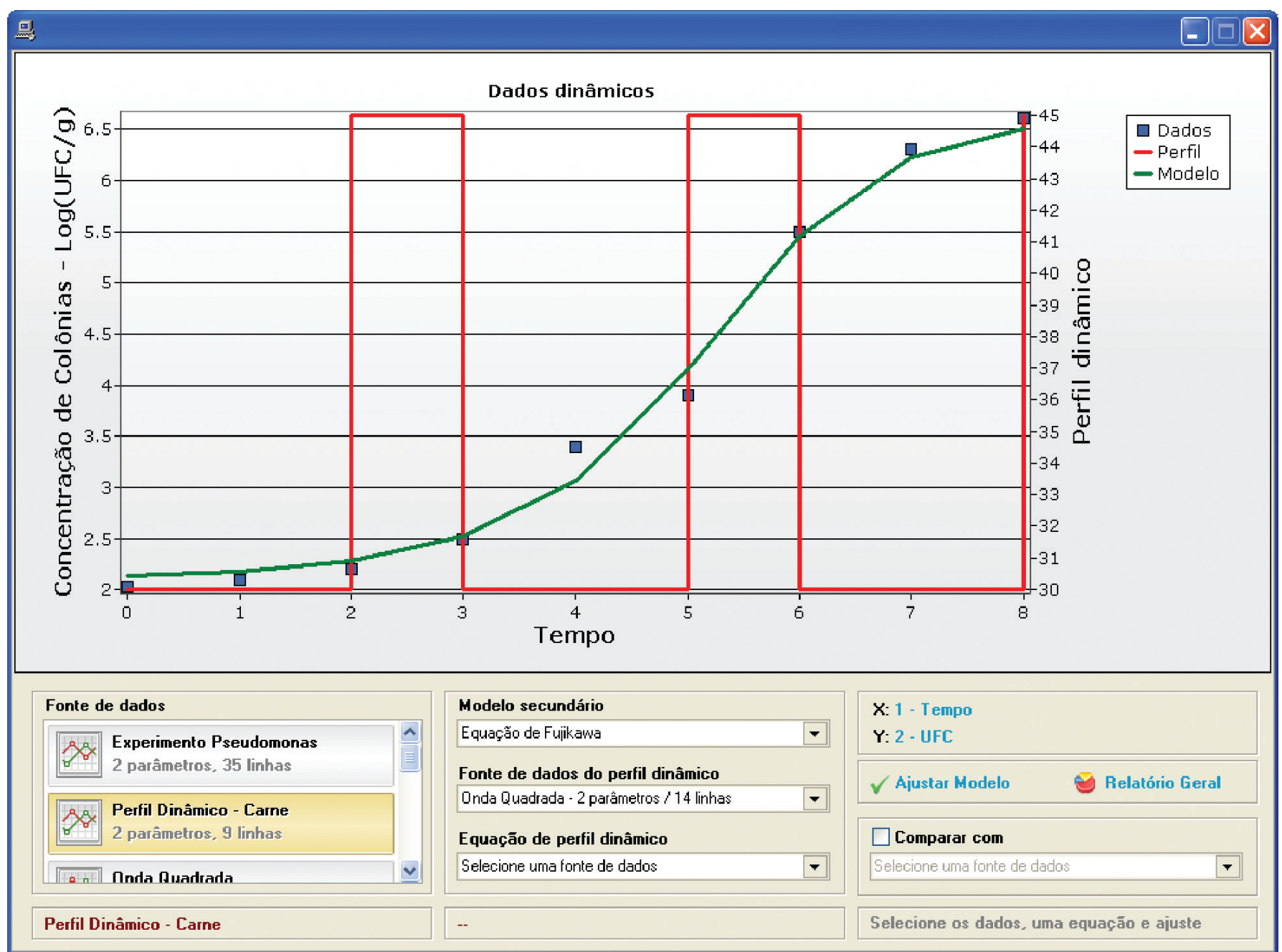

Figura 4. Modelos secundários de crescimento (eixo y, à esquerda), com um perfil de temperatura dinâmico (eixo y, à direita), em forma de onda quadrada, e uma curva de crescimento dinâmica em função do tempo (eixo x) no MicroFit.

Para calcular os valores dos parâmetros que melhor ajustam os modelos, o método utiliza o resultado de uma tentativa de ajuste como a própria estimativa inicial da próxima tentativa, acrescido de uma valor $\Delta$ que contém os pesos dos erros de cada parâmetro, tendendo a diminuir a diferença entre o valor fornecido pelo modelo e o valor dos dados coletados a cada iteração do algoritmo. Esse método possui elevada convergência para os principais modelos de crescimento encontrados na literatura (DANNENHAUER, 2010).

Para tornar a análise mais completa, os resíduos e as estatísticas intermediárias são apresentados ao usuário, bem como a possibilidade de se exportarem esses dados e utilizá-los em outros programas. Durante a comparação dos modelos, o peso de cada parâmetro é apresentado ao usuário, permitindo que seja decidido numericamente se o parâmetro tem influência no modelo, bem como a verificação do princípio da parcimônia.

O software descrito neste trabalho possui uma ferramenta ainda não abordada em outro programa do gênero: a possibilidade de desenvolver modelos utilizando-se funções matemáticas e parâmetros informados livremente pelo usuário, ou seja, o pesquisador pode inferir e testar seu próprio modelo, comparando-o com os já existentes.

É possível inserir no programa curvas não descritas na literatura para ajustes de dados microbiológicos, como a tangente hiperbólica, e compará-las com a equação diferencial de Fujikawa (FUJIKAWA et al., 2004) ou Baranyi-Roberts (CAYRÉ et al., 2007), por exemplo. Para isso, basta descrever no módulo de criação de modelos as equações, a exemplo de $y=a+b^{\star} \tanh \left(c^{\star} x\right)$, para a função hiperbólica, ou para a equação de Fujikawa, $y^{\prime}=w^{*}[1-y / \max (y)]^{*} \operatorname{pow}(1-[\min (y)-0.001] / y, c)$. Ao fim do processo é possível visualizar os parâmetros utilizados na execução, o grau de ajuste, a influência das variáveis e o erro médio do modelo ou, ainda, comparar dois modelos.

Em questão de segundos o pesquisador poderá obter resultados estatísticos dos modelos digitados como simples equações. De maneira semelhante torna-se 
MicroFit: um software gratuito para desenvolvimento e ajuste de modelos matemáticos de crescimento bacteriano SIQUEIRA, A. A. et al.

possível fazer ajuste de modelos secundários, de resolução ainda mais trabalhosa quando não se possui conhecimento e ferramentas apropriadas.

As soluções identificadas na Tabela 2 não descrevem as equações programadas internamente e quando descrevem formalmente, como o IPMP 2013, não realizam ajustes por minimização de erros, dificultando a comparação direta entre elas, entretanto, através do pacote de ajustes de funções não lineares, como o Solver ${ }^{\mathrm{TM}}$ e o software MatLab ${ }^{\circledR}$, notou-se que a resposta oferecida pelo programa é idêntica à dos softwares comparados até a sexta casa decimal. A Figura 5 ilustra um exemplo de criação de modelo (Tangente hiperbólica) e comparação com o modelo Logístico.

Essa característica tem como objetivo incentivar o desenvolvimento de novos modelos, cada vez mais precisos, de uma maneira fácil e segura. O desenvolvimento dessa ferramenta está fundamentado em um interpretador de funções codificado internamente no software, capaz de reconhecer uma cadeia de caracteres contendo uma função matemática que descreve ou não os dados experimentais através de variáveis, constantes e derivadas. Ao digitar a expressão que representa o modelo a ser testado, as estatísticas de um perfil de dados selecionado são calculadas e apresentadas para o usuário. Ressalta-se que todas as funções apresentadas possuem mecanismos de exportação de dados e representação gráfica.

A facilidade de trabalhar com equações e dados dentro do programa desenvolvido abstrai a utilização de métodos numéricos de ajuste, permitindo que pesquisadores não familiarizados com esses métodos possam ajustar curvas e desenvolver novos

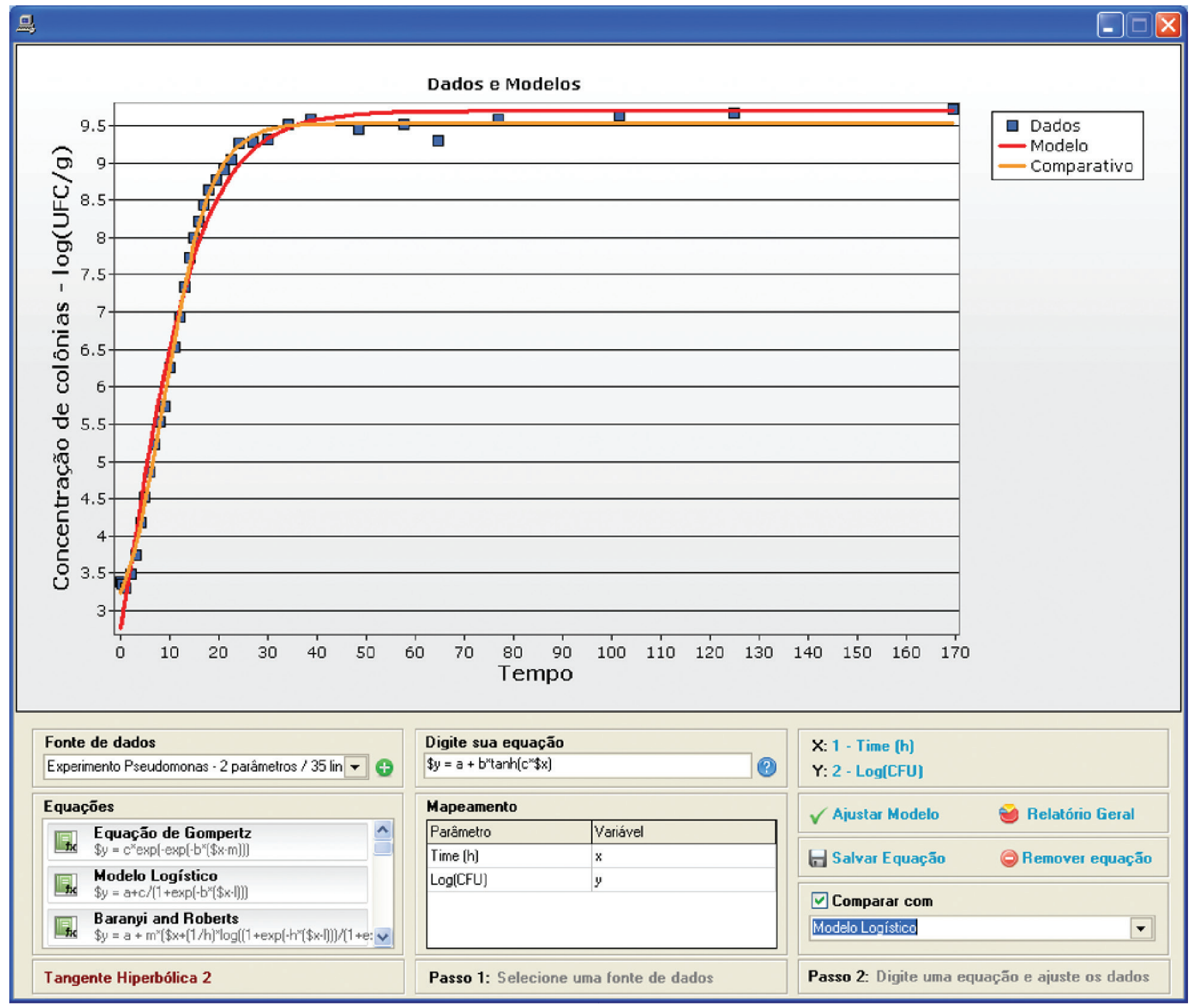

Figura 5. Interface para desenvolvimento de novos modelos teóricos e sintaxe de uso das funções disponíveis e aceitas pelo software MicroFit, com comparação de modelos. 
MicroFit: um software gratuito para desenvolvimento e ajuste de modelos matemáticos de crescimento bacteriano SIQUEIRA, A. A. et al.

modelos focando na explicação microbiológica dos parâmetros informados, sem a necessidade de realizar análises matemáticas complexas que podem impedir o empreendimento. Com a curva ajustada é possível conhecer a taxa de crescimento de um micro-organismo, o efeito do ambiente sobre ele, estabelecer a vida útil de um produto e tomar decisões com base nos dados apresentados pelo software.

Apesar de este trabalho ter citado apenas o uso em ajuste de modelos microbiológicos de crescimento, o software poderá ser utilizado com as mesmas ferramentas para ajustes genéricos de funções e modelos não necessariamente ligados à microbiologia preditiva. As ferramentas presentes nesse programa, a facilidade de uso, a possibilidade de montar e testar modelos rapidamente, somadas ao fato de o programa ser gratuito e independente de outras ferramentas, tornam-no mais atrativo, competitivo e distinto das soluções analisadas anteriormente.

O programa desenvolvido não tem como objetivo substituir nenhuma das soluções analisadas neste artigo, mas sim de complementá-las e oferecer aos pesquisadores de modelos preditivos uma ferramenta para trabalhar com ajuste de modelos microbiológicos e tomada de decisões rápidas. O programa encontra-se em versão estável e novos estudos com intuito de adicionar novas ferramentas, métricas de comparação e exemplos estão em desenvolvimento para implementação de uma versão futura. Após os trâmites de seu registro no INPI, o programa poderá ser baixado gratuitamente no link http://www.univasf.edu.br/ cpgea/arquivos/.

\section{Conclusão}

O MicroFit mostra-se como uma alternativa viável de software gratuito voltado para o ajuste de dados experimentais a modelos preditivos teóricos fundamentado em equações não lineares, capaz de abstrair a complexidade matemática envolvida no processo sem limitar o pesquisador.

Além de realizar o ajuste automático dos dados experimentais a modelos de crescimento teórico de bactérias, permite a criação, montagem e ajuste de novo modelos de uma maneira fácil e acessível aos profissionais da microbiologia. As falhas encontradas nos programas analisados foram corrigidas através da criação de um programa gratuito, robusto e fácil de utilizar, composto de ferramentas únicas, que permitem ao usuário desenvolver modelos cada vez mais precisos. Espera-se que essa ferramenta possa contribuir para a melhoria da segurança e das estimativas do tempo de vida de produtos, bem como para outros empreendimentos, através da criação de modelos e das funções para ajuste de dados.

\section{Agradecimentos}

À Fapesp, pela bolsa de estudos concedida ao primeiro autor.

\section{Referências}

ARROYO-LÓPEZ, F. N.; BAUTISTA-GALLEGO, J.; GARRIDOFERNÁNDEZ, A. Role of predictive microbiology in food preservation. In: BHAT, R.; ALIAS, A. K.; PALIYATH, G. Progress in food preservation. West Sussex: Wiley-Blackwell, 2012. cap. 19.

ARROYO-LÓPEZ, F. N.; BAUTISTA-GALLEGO, J.; GARCÍAGIMENO, R. M.; GARRIDO-FERNÁNDEZ, A. Predictive microbiology: a valuable tool in food safety. In: BHAT, R.; GOMEZ-LOPEZ, V. M. Practical food safety: contemporary issues and future directions. West Sussex: Wiley Blackwell, 2014. cap. 25

BRASIL. Ministério da Saúde. Secretaria de Vigilância em Saúde. Vigilância epidemiológica das doenças transmitidas por alimentos no Brasil, 1999-2004. Boletim Eletrônico Epidemiológico, Brasília, v. 5, n. 6, 2005. Disponível em: <http:// bvsms.saude.gov.br/bvs/periodicos/boletim_eletronico_epi_ ano05_n06.pdf> Acesso em: 9 maio 2014.

CASTRO, M. F. P. P. M.; SCHMIDT, F. L.; PENTEADO, A. L. Growth of Salmonella Enteriditidis and Listeria Monocytogenes in melon pulp: predictive modelling and evaluation of model performance. Brazilian Journal of Food Technology, Campinas, v. 10, n. 3, p. 176-182, 2007.

CAYRÉ, M. E.; VIGNOLO, G. M.; GARRO, O. A. Selección de un modelo primario para describir la curva de crecimiento de bacterias lácticas y brochothrix thermosphacta sobre emulsiones cárnicas cocidas. Información Tecnológica, La Serena, v. 18, n. 3, p. 23-29, 2007

CENTERS FOR DISEASE CONTROL AND PREVENTION - CDC. Estimating foodborne Illness: an overview. Atlanta: CDC, 2011. Disponível em: <http://www.cdc.gov/foodborneburden/ estimates-overview.html> Acesso em: 9 maio 2014.

COMBASE. Disponível em: <http://www.combase.cc/index.php/ en/downloads>. Acesso em: 19 abr. 2014.

DANNENHAUER, C. E. Desenvolvimento de um aplicativo computacional para microbiologia preditiva. 2010. $147 \mathrm{f}$. Dissertação (Mestrado em Engenharia de Alimento)-Centro Tecnológico, Universidade Federal de Santa Catarina, Florianópolis, 2010.

FUJIKAWA, H.; KAI, A.; MOROZUMI, S. A new logistic model for E. coli growth at constant and dynamic temperatures. Food Microbiology, Summit-Argo, v. 21, n. 5, p. 501-509, 2004.

GAVIN, H. P. The Levenberg-Marquardt method for nonlinear least squares curve-fitting problems. Durham: Duke University, 
MicroFit: um software gratuito para desenvolvimento e ajuste de modelos matemáticos de crescimento bacteriano SIQUEIRA, A. A. et al.

2013. 17 p. Disponível em: <http://people.duke.edu/ hpgavin/ ce281/lm.pdf> Acesso em: 18 jun. 2014.

GEERAERD, A. H.; VALDRAMIDIS, V. P.; VAN IMPE, J. F. GInaFiT, a freeware tool to assess non-log-linear microbial survivor curves. International Journal of Food Microbiology, Wyndmoor, v. 102, p. 95-105, 2005.

HUANG, L. Dynamic computer simulation of Clostridium perfringens growth in cooked ground beef. International Journal of Food Microbiology, Wyndmoor, v. 87, p. 217-227, 2003.

MCCLURE, P.; AMÉZQUITA, A. Predicting the behaviour of microorganisms in chilled foods. In: BROWN, M. Chilled foods: a comprehensive guide. 3rd ed. Abington: Woodhead Publishing, 2009. cap. 17. p. 500-511.

MOREIRA, S. A. Alimentação e comensalidade: aspectos históricos e antropológicos. Ciência e Cultura, Campinas, v. 62, n. 4, 2010. Disponível em: <http://cienciaecultura.bvs. $\mathrm{br} /$ scielo. php?script=sci_arttext\&pid=S0009-67252010000 400009\&lng=en\&nrm=iso >. Acesso em: 18 jun. 2014.

NAKASHIMA, S. M. K.; ANDRÉ, D. S.; FRANCO, B. D. G.M. Revisão: aspectos básicos da microbiologia preditiva. Brazilian Journal of Food Technology, Campinas, v. 3, p. 41-51, 2000.

PMM LAB - PMM. Predictive microbial modeling. Disponível em: <http://sourceforge.net/p/pmmlab/wiki/Links>. Acesso em: 18 abr. 2014.

SARMENTO, C. M. P. Modelagem do crescimento microbiano e avaliação sensorial no estudo da vida de prateleira de mortadela e linguiça defumada em armazenamento isotérmico e não isotérmico. 2006. 145 f. Dissertação (Doutorado em Engenharia Química)-Universidade Federal de Santa Catarina, Florianópolis, 2006.
SWINNEN, I. A. M.; BERNAERTS, K.; DENS, E. J. J.; GEERAERD, A. H.; VAN IMPE, J. F. Predictive modelling of the microbial lag phase: a review. International Journal of Food Microbiology, Wyndmoor, v. 94, p. 137-159, 2004.

TELEKEN, J. T.; ROBAZZA, W. S.; GOMES, G. A. Mathematical modeling of microbial growth in milk. Ciência e Tecnologia de Alimentos, Campinas, v. 31, n. 4, p. 891-896, 2011. Disponível em: <http://www.scielo.br/pdf/cta/v31n4/10.pdf>.

UC FOOD SAFETY. Pathogen modeling programs. California: Division of Agriculture and Natural Resources, University of California. Disponível em: <http://ucfoodsafety.ucdavis.edu/ Food_Safety_Links/Pathogen_Modeling_Programs/>. Acesso em: 18 abr. 2014.

VALLE, K. N. F. Métodos numéricos de Euler e Runge-Kutta. 2012. 40 f. Monografia (Especialização em Matemática)Universidade Federal de Minas Gerais, Belo-Horizonte, 2012. Disponível em: <http://www.mat.ufmg.br/ espec/Monografias_ Noturna/ Monografia_KarineNayara.pdf>. Acesso em: 18 jun. 2014.

VALSECHI, O. C. Microbiologia dos alimentos. Araras: Centro de Ciências Agrárias, Departamento de Tecnologia Agroindustrial e Socioeconomia Rural, Universidade Federal de São Carlos, 2006. Disponível em: <http://www.cca.ufscar. $\mathrm{br} /$ vico/Microbiologia\%20dos\%20Alimentos.pdf>. Acesso em: 12 maio 2014.

ZHOU, K.; GUI, M.; LI, P.; XING, S.; CUI, T.; PENG, Z. Effect of combined function of temperature and water activity on the growth of Vibrio harveyi. Brazilian Journal of Food Technology, Campinas, v. 43, n. 4, p. 1365-1375, 2012. 